\section{Konsens über fokale Brachytherapie}

Eine Fokussierung der Brachytherapie auf befallene Bereiche der Prostata soll die Nebenwirkungen des üblichen Vorgehens reduzieren. Welche Patienten infrage kommen und wie vorzugehen ist, darüber gibt es nun einen Konsens.

\begin{abstract}
E xperten schätzen, dass bis zu zwei
C Drittel aller Patienten mit einer fokalen Brachytherapie mit niedrigen Strahlendosen behandelt werden könnten. Dabei wird besonders der betroffene Seitenlappen mit Seeds ins Visier genommen. Allerdings existierte bis dato kein einheitliches Therapieprotokoll. Dazu hat eine Expertengruppe nun ein Konsensuspapier vorgelegt. Darin ist unter anderem beschrieben, welche Patienten mit fokaler Brachytherapie behandelt werden sollten und wie das technische Vorgehen auszusehen hat:

- Lebenserwartung > 10 Jahre;

-PSA-Wert $\leq 15 \mathrm{ng} / \mathrm{ml}$;

_multiparametrische MRT vor der Biopsie;

_bilaterale, Matrix-geleitete Biopsie der

kartierten Prostata (Mapping-Biopsie)

im 5-mm-Raster;
\end{abstract}

_einseitige Erkrankung mit Läsionsvolumen $\leq 0,5 \mathrm{ml}$, maximale Länge $1 \mathrm{~cm}$; kontralateral keine oder keine klinisch signifikante Erkrankung (Tumorlänge $\leq 3 \mathrm{~mm}$ );

_Gleason-Score der Indexläsion 6-7 (3 $+4)$;

- Tumorstadium $\leq 2 \mathrm{~b}$;

_-Prostatavolumen $\leq 60 \mathrm{ml}$.

Therapiert wird mit Jod-125-Seeds niedriger Aktivität. $\mathrm{Zu}$ berücksichtigen sind wie bei herkömmlicher Brachytherapie Rektal- und Urethraldosen. Weitere Risikoorgane sind Bulbus penis und neurovaskuläres Bündel am Apex der Drüse.

Noch nicht endgültig geklärt ist die Dosierung:

- Brachytherapie der befallenen Drüsenhälfte mit 145 Gy bei einseitiger Erkrankung oder

\section{Bestrahlung gegen Blasenkrebs mit Chemotherapie wirksamer}

Bei muskelinvasivem Blasenkrebs lassen sich mittels Radiotherapie nur bescheidene Erfolge erzielen. In der Studie BC2001 (Bladder Cancer 2001) wurde deswegen überprüft, ob eine Kotherapie mit Fluorouracil und Mitomycin $C$ das Behandlungsergebnis verbessern kann.

$\mathrm{R}$ andomisiert wurden in dieser Studie 360 Patienten mit invasivem Blasenkrebs im Stadium T2, T3 oder T4a ohne Lymphknotenbeteiligung oder Metastasen der Radiotherapie oder der Chemoradiotherapie zugeteilt. Für die Radiotherapie konnten 55 Gy in 20 Fraktionen über vier Wochen oder 64 Gy in 32 Fraktionen über 6,5 Wochen appliziert werden. Fluorouracil (500 mg/m² Körperoberfläche pro Tag) wurde als kontinuierliche Infusion zwischen den Fraktionen 1-5 und 16-20 für insgesamt zehn Tage und Mitomycin $\mathrm{C}\left(12 \mathrm{mg} / \mathrm{m}^{2}\right)$ als i.v.-Bolus an Tag 1 gegeben. Nach der Behandlung wurden die Patienten im Median 70 Monate weiterverfolgt.

Die Chance, ohne lokoregionales Rezidiv zu überleben, war in der Gruppe mit
Chemoradiotherapie signifikant besser: Nach zwei Jahren waren noch $67 \%$ der Patienten rezidivfrei - im Vergleich zu $54 \%$ in der Bestrahlungsgruppe. Besonders deutlich zeigte sich der Nutzen der Chemotherapie in Bezug auf das Wiederauftreten von invasiven Karzinomen: Davon waren $18 \%$ der Chemoradiotherapie-Patienten betroffen, unter alleiniger Radiotherapie waren es $32 \%$. Bei den $\mathrm{Pa}$ tienten mit Chemotherapie wurden tendenziell, aber nicht signifikant weniger Zystektomien durchgeführt (11,4 vs. $16,8 \%)$. Das Gesamtüberleben nach fünf Jahren war ebenfalls nicht signifikant verschieden: Von den kombiniert behandelten Patienten waren noch $48 \%$, von den ausschließlich bestrahlten Patienten noch $35 \%$ am Leben. _zusätzlich Applikation einer niedrigen Dosis in der gesamten Drüse bei einseitiger Erkrankung und klinisch nicht signifikanter Erkrankung kontralateral.

Fazit: Das Konsensuspapier gibt eine erste Handreichung dazu, wie für die fokale Therapie geeignete Patienten auszuwählen sind und wie das Verfahren technisch umzusetzen ist. Präzisere Vorgaben zur Dosierung sollen demnächst folgen, ebenso der Plan für eine randomisierte PhaseII-Studie.

Robert Bublak

Langley $\mathrm{S}$ et al. Report of a consensus meeting on focal low dose rate brachytherapy for prostate cancer. BJU Int. 2012 Feb;109 Suppl 1:7-16. doi: 0.1111/j.1464-410X.2011. 10825.x.

In der September-Ausgabe 2012 von IM FOCUS ONKOLOGIE erscheint ein ausführlicher CME-Beitrag zu den Formen der fokalen Therapie beim Prostatakarzinom.

Die zusätzliche Chemotherapie erwies sich als relativ gut verträglich. Nebenwirkungen vom Schweregrad Grad 3 oder 4 traten während der Behandlung geringfügig, aber nicht signifikant häufiger auf (36,0 vs. $27,5 \%)$. Die späte Toxizität wurde durch die intensivierte Therapie nicht erhöht (8,3 vs. $15,7 \%)$.

Fazit: Durch die Hinzunahme der Chemotherapie mit Fluorouracil und Mitomycin C wurden in der Studie lokale Rezidive um ein Drittel und invasive Rezidivkarzinome fast um die Hälfte reduziert. Die Blasenfunktion wurde dadurch nicht stärker geschädigt, und nur selten musste doch noch eine Zystektomie durchgeführt werden. Dies dürfte vor allem für Blasenkrebspatienten mit hohem Operationsrisiko wichtige Erkenntnisse sein.

Beate Schumacher

James ND et al. Radiotherapy with or without chemotherapy in muscle-invasive bladder cancer. N Engl J Med. 2012;366(16):1477-88. - Editorial: William U et al. Old drugs, new purpose - bladder cancer turning a corner. Ibidem 1540-1. 